\title{
COMPLEXES WITH THE DISAPPEARING CLOSED SET PROPERTY
}

\author{
VYRON M. KLASSEN
}

\begin{abstract}
A topological space $X$ is said to have the disappearing closed set (DCS) property if and only if for every proper closed subset $C$ there is a sequence of homeomorphisms $\left\{h_{i}\right\}, i=1,2,3, \cdots$, of $X$ onto $X$, and a decreasing sequence of open subsets $\left\{U_{i}\right\}$, $i=1,2,3, \cdots$, of $X$ such that $\bigcap_{i=1}^{\infty} U_{i}=\varnothing$ and $h_{i}(C) \subseteq U_{i}$. THEOREM. A finite simplicial $n$-complex is an $n$-manifold if and only if it has the DCS property.
\end{abstract}

A topological space $X$ is said to have the disappearing closed set (DCS) property if and only if for every proper closed set $C \subset X$ there is a decreasing family of open sets $\left\{U_{i}\right\}, i=1,2, \cdots$, in $X$ such that $\bigcap_{i=1}^{\infty} U_{i}=\varnothing$, and a sequence of homeomorphisms $\left\{h_{i}\right\}_{i=1}^{\infty}$ of $X$ onto $X$ such that $h_{i}(C) \subseteq$ $U_{i}, i=1,2, \cdots$.

This definition was motivated by an attempt to study ideas related to invertible spaces ([1], [2]). Examples of spaces with the DCS property are the $n$-sphere, open $n$-cell, torus and open annulus. A disconnected example is $(0,1) \cap$ \{rationals\}. It can be proved that any disconnected DCS space must have infinitely many components, and that the product of two DCS spaces will also have the DCS property [3]. It is proved here that, under certain restrictions, the union of two spaces each of which has the DCS property will have the DCS property. This is then used to prove that a finite simplicial $n$-complex is an $n$-manifold if and only if it has the DCS property.

THEOREM 1. Let $X$ be a space with two intersecting open subspaces $C$ and $D$ each of which has the DCS property, such that for any proper closed subset of $C$ (or D, resp.), the sequences of open sets $\left\{U_{i}\right\}_{i=1}^{\infty}\left(\left\{V_{i}\right\}_{i=1}^{\infty}\right)$ and homeomorphisms $\left\{h_{i}\right\}_{i=1}^{\infty}\left(\left\{k_{i}\right\}_{i=1}^{\infty}\right)$ may be taken to have the following properties:

(a) $h_{i}\left(k_{i}\right)$ may be extended to a homeomorphism of $X$ onto $X$ that is the identity on $X-C(X-D)$.

(b) There is a positive integer $M(N)$ such that $C \cap\left(D-\bar{U}_{M I}\right) \neq \varnothing$ $\left(D \cap\left(C-\bar{V}_{.}\right) \neq \varnothing\right)$. (The closure is with respect to $C \cup D$.)

Presented to the Society, April 10, 1971; received by the editors March 3, 1971. AMS 1970 subject classifications. Primary 54D99, 57A05, 57A10, 57A14.

Key words and phrases. $n$-manifold, $n$-complex, disappearing closed set property.

(C) American Mathematical Society 1973 
Then $C \cup D$ has the DCS property, with a sequence of homeomorphisms $\left\{\varphi_{i}\right\}_{i=1}^{\infty}$ such that $\varphi_{i}$ may be extended to a homeomorphism of $X$ onto $X$ that is the identity on $X-(C \cup D)$.

Proof. Let $A$ be a proper closed set in $C \cup D$, with respect to the relative topology. If $A \cap C=\varnothing$ or $A \cap D=\varnothing$, there is nothing to prove. Without loss of generality, it may be assumed that $A \cap C$ is a proper closed subset of $C$. From the DCS property on $C$ and the hypothesis of the theorem, there is a sequence $\left\{U_{i}\right\}_{i=1}^{\infty}$ of open sets in $C$ such that $U_{i+1} \subseteq U_{i}$ and $\bigcap_{i=1}^{\infty} U_{i}=\varnothing$, and a sequence of homeomorphisms $\left\{h_{i}\right\}_{i=1}^{\infty}$ of $X$ onto $X$ such that $h_{i}(A \cap C) \subseteq U_{i}$ and $h_{i} \mid X-C$ is the identity, for all $i$.

Let $M$ be the positive integer such that $C \cap\left(D-\bar{U}_{M}\right) \neq \varnothing .\left[\bar{U}_{M I} \cap D\right] \cup$ $(D-C)=B$ is a closed proper subset of $D$, so there is a sequence $\left\{k_{i}\right\}_{i=1}^{\infty}$ of homeomorphisms of $X$ onto $X$, the identity on $X-D$ and a decreasing sequence $\left\{V_{i}\right\}_{i=1}^{\infty}$ of open sets in $D$ such that $k_{i}(B) \subseteq V_{i}$, for all $i$, and $\bigcap_{i=1}^{\infty} V_{i}=\varnothing$.

Let $\varphi_{i}=k_{i} \circ h_{M+i}$, and let $W_{i}=U_{M+i} \cup V_{i} . W_{i}$ is open for all $i$. Now $h_{. M+i}(A) \subseteq B \cup\left[U_{M+i} \cap(C-D)\right]$, so $\varphi_{i}(A) \subseteq k_{i}\left\{B \cup\left[U_{M+i} \cap(C-D)\right]\right\} \subseteq W_{i}$. (Note that $\varphi_{i} \mid C-D=h_{M+i}$, and $\varphi_{i} \mid D-C=k_{i}$.) Also, $W_{i+1} \subseteq W_{i}$, since $U_{\lambda I+i+1} \subseteq U_{\lambda+i}$ and $V_{i+1} \subseteq V_{i}$, and $\bigcap_{i=1}^{\infty} W_{i}=\varnothing$. Finally $\varphi_{i} \mid X-(C \cup D)$ is the identity, so the theorem is proved.

THEOREM 2. Let $D$ be a closed $n$-cell, with interior $C$. Then $C$ has the DCS property, and the sequences of DCS homeomorphisms may be chosen to extend to the identity on $D-C$.

Proof. Let $A$ be a closed proper subset of $C$ in the relative topology for $C$. There is a point $y \in C-A$ which is contained in an open set in $C-A$. Since $D$ is a closed $n$-cell, it may be expressed as a closed cone neighborhood of $y$, say $[0,1) \times S^{n-1} \cup y$. By radial projection on this cone, holding $y$ and $D-C$ pointwise fixed, it is possible to homeomorphically move $A$ into $[0,1 / m) \times S^{n-1}$, for all $m$. If we then take $U_{m}=(0,1 / m) \times S^{n-1}$, the theorem is proved.

Corollary (TO THE PROOF OF TheOREM 2). If $B$ is an open n-cell such that $C \cap B \neq \varnothing$, there is a $p$ such that $C \cap\left(B-\bar{U}_{p}\right) \neq \varnothing$.

THEOREM 3. A finite, connected simplicial n-complex is an n-manifold if and only if it has the DCS property.

Proof. The set of points where a complex is not locally Euclidean is closed and invariant (and a proper subset). It must be empty by the DCS property. Thus, the complex is an $n$-manifold.

Now, suppose the $n$-complex $X$ is an $n$-manifold. Let $S_{1}, S_{2}, \cdots, S_{n}$ be the (closed) stars of the $n$-vertices in $X$. Let $U_{i}$ be the interior of $S_{i}$, 
$i=1, \cdots, n$. Note that $X=\bigcup_{i=1}^{n} U_{i}$. Each $U_{i}$ is an open $n$-cell, since $X$ is a manifold.

Let $A$ be a proper closed subset of $X$. There is a $j$ such that $A \cap U_{j}$ is a proper relatively closed subset of $U_{j}$. Let $U_{j}=V_{1}$, and $V_{k}=U_{i}$ with $i$ such that $U_{i} \cap V_{k-1} \neq \varnothing, V_{k} \nsubseteq \bigcup_{j}^{k-1} V_{j}$.

Now by Theorem 2, the DCS homeomorphisms for $V_{k}$ may be taken to be the identity on $\bar{V}_{k}-V_{k}=\mathrm{Bd} V_{k}$, so they may be taken to be the identity on $X-V_{k}$. By Theorems 1 and $2, V_{1} \cup V_{2}$ has the DCS property.

Similar reasoning shows that $\bigcup_{k=1}^{i} V_{k}$ has the DCS property, $i=3, \cdots$, $n$, since $\bigcup_{k=1}^{i-1} V_{k}$ and $V_{i}$ satisfy conditions (a) and (b) in Theorem 1. Thus, the theorem is proved.

\section{REFERENCES}

1. P. H. Doyle and J. G. Hocking, A characterization of Euclidean n-space, Michigan Math. J. 7 (1960), 199-200. MR 22 \#12515.

2. - Invertible spaces, Amer. Math. Monthly 68 (1961), 959-965. MR 24 \#A1711.

3. V. M. Klassen, The DCS property, Pacific J. Math. (to appear).

Department of Mathematics, California State College, Fullerton, California 92631 